\title{
Effect of Novel Nanocomposite Materials for Enhancing Performance of Thin Film Transistor TFT Model
}

\author{
Youssef Mobarak***, Moamen Atef** \\ * Department of Electrical Engineering, Faculty of Engineering, Rabigh, King Abdulaziz University, Saudi Arabia \\ ** Department of Electrical Engineering, Faculty of Energy Engineering, Aswan University, Egypt
}

\section{Article Info \\ Article history: \\ Received Dec 12, 2015 \\ Revised Feb 18, 2016 \\ Accepted Feb 27, 2016}

\section{Keyword:}

COMS

Nanocomposite gate dielectric

Sidewall spacer

Thin Film Transistor (TFT)

Threshold voltage

\begin{abstract}
The potential impact of high permittivity gate dielectrics on thin film transistors short channel and circuit performance has been studied using highly accurate analytical models. In addition, the gate-to-channel capacitance and parasitic fringe capacitances have been extracted. The suggested model in this paper has been increased the surface potential and decreased the threshold voltage, whenever the conventional silicon dioxide gate dielectric is replaced by high- $\mathrm{K}$ gate dielectric novel nanocomposite $\mathrm{PVP} / \mathrm{La}_{2} \mathrm{O}_{3} \mathrm{~K}_{\mathrm{ox}}=25$. Also, it has been investigated that a decrease in parasitic outer fringe capacitance and gate-to-channel capacitance, whenever the conventional silicon nitride is replaced by low- $\mathrm{K}$ gate sidewall spacer dielectric novel nanocomposite $\mathrm{PTFE} / \mathrm{SiO}_{2} \mathrm{~K}_{\mathrm{sp}}=2.9$. Finally, it has been demonstrated that using low-K gate sidewalls with high-K gate insulators can be decreased the gate fringing field and threshold voltage. In addition, fabrication of nanocomposites from polymers and nano-oxide particles found to have potential candidates for using it in a wide range of applications in low cost due to low process temperature of these nanocomposites materials.
\end{abstract}

Copyright $@ 2016$ Institute of Advanced Engineering and Science. All rights reserved.

\section{Corresponding Author:}

Youssef Mobarak,

Departement of Electrical Engineering,

Faculty of Energy Engineering, Aswan University,

81528, Aswan, Egypt.

Email: ysoliman@aswu.edu.eg

\section{INTRODUCTION}

Integrated Circuits IC's can include as many as hundred million transistors or more. As increased gate leakage is one major limiting factor on aggressive scaling effective gate-dielectric thickness will require alternative materials with higher permittivity's Kox and greater physical thicknesses, a factor of (Kox/KSiO2) to prevent direct gate tunneling [1], [3]. However, the use of a high-K gate material may result in dielectric thicknesses comparable to the device gate length, fringing fields from the gate to the source/drain regions compromising the short-channel performance increasing. Kumar and Chaudhry [4] have earlier developed a model for the threshold voltage for dual material gate SOI-MOSFETs. Our suggested model for the threshold voltage for the single nanocomposite material gate SOI-MOSFETs by including the effect of the internal fringe capacitance on the threshold voltage, which can be easily, solved using a few iterations. In order to accurately characterize TFT devices, the threshold voltage must be well formulated. Our suggested model with novel nano composite material PVP/La2O3 with high dielectric constant enhances the performance of MOSFETs. By increasing in the surface potential and decreasing the threshold voltage. It has been demonstrated a suitable low-permittivity low-K gate sidewall spacer dielectric novel nanocomposite $\mathrm{PTFE} / \mathrm{SiO} 2, \mathrm{Ksp}=2.9$, which can replace $\mathrm{Si3N} 4, \mathrm{Ksp}=7.5$ [4-11].

The energy of the band edge is the threshold condition for conduction in the TDT (some authors redefine the TDT model as the mobility edge model [12] by modifying the tail shape). It can be shown that 
the expressions for $\mu$ o derive din the TDT [13] and VRH [14] theories differ by two constant multipliers [15]. This setting violates the conditions for proper use of the infinite integration limit in the gamma function in the theoretical derivations in [13], [14]. This problem was recently identified in [16]. The variety of models for OTFTs is large, ranging from simple phenomenological models based on similarities to crystal FETs, to finite-element models of the TFT structure [17], and from the adaptation of a-TFT charge sheet models [18] to limiting models [19]. Each of these models has advantages and disadvantages, addressing different issues such as complexity, accuracy, repeatability, physics, characterization techniques, or convergence. On the other hand, the object of the models is the same accurate prediction of the electrical characteristics of OTFTs. Recently, the fringing capacitance was present through the conformal mapping of potential space. Fringing Induced Barrier Lowing FIBL is the effect of charges induced by the fringing capacitances, a threshold voltage case including the fringing capacitance effect is studied and the influence of gate dielectric on threshold voltage is discussed [20-27].

\section{THIN FILM TRANSISTOR TFT MODEL}

A model cross-sectional of an SOI-MOSFET with a high-Kok gate dielectric is shown in Figure 1(a), with the fringing field lines from the bottom of the gate electrode to the drain and source regions. For simplicity, that circular field lines as shown in Figure 1(b), and to the approach used in studies for the other components of parasitic capacitance [7], [8]. The infinitesimal capacitances in the high-Kok and spacer regions, respectively can be written as:

$$
d C_{1}=\frac{K_{o x} W d y}{\left(\frac{\pi}{2}\right) y}, \text { and } d C_{2}=\frac{K_{s p} W d y}{\left(\frac{\pi}{2}\right)\left(t_{o x}-y\right)}
$$

Where $\mathrm{K}_{\mathrm{ok}}$ is the permittivity of the gate dielectric, $\mathrm{K}_{\mathrm{SP}}$ is the permittivity of spacer material, $\mathrm{W}$ is the width of the transistor structure, and tok is the gate-dielectric thickness. Since $\mathrm{dC}_{1}$ and $\mathrm{dC}_{2}$ are in series, the net infinitesimal capacitance can be written as:

$$
d C=\frac{d C_{1} d C_{2}}{d C_{1}+d C_{2}}=\frac{2 K_{o x} K k_{s p} W d y}{\pi\left(K_{o x} t_{o x}+K_{s p} y-K_{o x} y\right)}
$$

The total internal fringe capacitance can be obtained by integrating (2) over the gate-dielectric thickness as fringing:

$$
\begin{aligned}
& C_{\text {bottom }}=\int_{0}^{t_{o x}} \frac{2 K_{o x} K_{s p} W d y}{\pi\left(K_{o x} t_{o x}+K_{s p} y-K_{o x} y\right)} \\
& C_{\text {bottom }}=\frac{2 k_{o x} K_{S p} W}{\pi\left(K_{o x}-K_{s p}\right)} \ln \left(\frac{K_{o x}}{K_{s p}}\right):
\end{aligned}
$$

From the entire perimeter of the bottom edge of the gate electrode as:

$$
C=\frac{\left((2-\ln 4) K_{O X}\right)}{2 \pi}
$$

For $K_{o x=} K_{s p}$, and $\frac{t_{o x}}{L_{g}} \ll 1$

Where: $L_{g}$ is the gate length. 


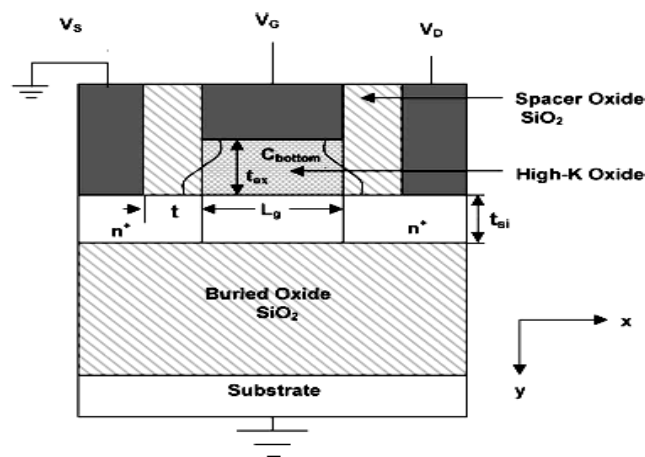

Figure 1(a). Cross-sectional of an SOI-MOSFET, Internal parasitic fringe capacitance

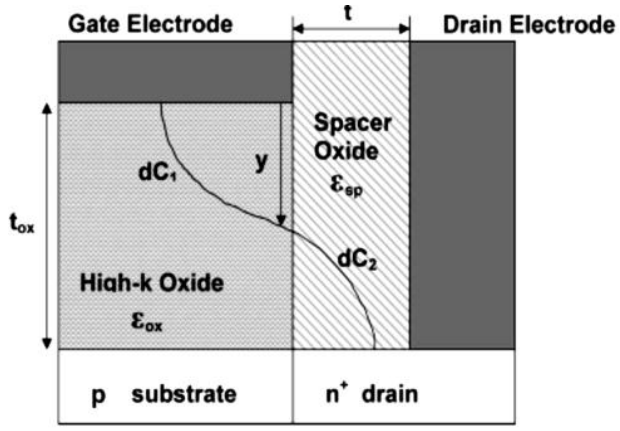

Figure 1(b). Cross-sectional of an SOI-MOSFET, Fringing field bottom of the gate electrode

The total fringe capacitance can be obtained by Equation (4), and the perimeter of the bottom edge of the gate electrode [2]. Since the account for electric field lines fringing from the bottom edge of the gate to either the source or the drain region only, the internal fringe capacitance can be written as:

$$
C_{\text {bottom }}=\frac{\left((2-\ln 4) K_{o x} W\right)}{2 \pi} \cong \frac{(0.3) K_{o x} W}{\pi}
$$

For $K_{o x=} K_{s p}$, and $\frac{t_{o x}}{L_{g}} \ll 1$

It can be seen that Equ. (3) reduces to Equation (5) in the limit $\mathrm{K}_{\mathrm{ok}} \rightarrow \mathrm{K}_{\mathrm{SP}}$ but for a constant factor. This difference arises because of the assumption of the fringing electric field lines is being circular while deriving Equation (3). thus, multiplied by the above factor equally 0.15 to obtain:

$$
\mathrm{C}_{\text {bottom }}=\frac{(0.3) \mathrm{K}_{\mathrm{ox}} \mathrm{K}_{\mathrm{sp}} \mathrm{W}}{\pi\left(\mathrm{K}_{\mathrm{ox}}-\mathrm{K}_{\mathrm{sp}}\right)} \ln \left(\frac{\mathrm{K}_{\mathrm{ox}}}{\mathrm{K}_{\mathrm{sp}}}\right)
$$

These expression reduces to Equ. (5) the limit $\mathrm{K}_{\mathrm{o}} \mathrm{k} \rightarrow \mathrm{K}_{\mathrm{SP}}$, and hence Equ. (6), that the separation between the electrodes is very small in comparison to the length of the electrodes [10]. This is certainly not the case in short channel high-k dielectric SOI-MOSFETs. Further modified to represent the true picture, and the fringing field from the gate to the source/drain regions increases as a function of $\left(t_{o x} / L_{g}\right)$. This is the effect of the increased crowding of field lines in the spacer region for large gate-dielectric thicknesses, for tox comparable to $\mathrm{L}_{\mathrm{g}}$, the fringe capacitance in the spacer region is more than what has been assumed while Equ. (6). to account for this, an effective spacer dielectric constant as:

$$
K_{s p}^{\prime}=\left(1+\frac{t_{o x}}{L_{g}}\right) \mathrm{K}_{\mathrm{sp}}
$$

Substituting (7) in place of $\mathrm{K}_{\mathrm{SP}}$ in (6), then obtain the final expression for the parasitic internal fringe capacitance as:

$$
\mathrm{C}_{\text {bottom }}=\frac{(0.3) \mathrm{k}_{\mathrm{eff}} \mathrm{W}}{\pi}
$$

Where: $k_{e f f}=\frac{K_{o x} K_{s p}^{\prime}}{K_{o x}-K_{s p}^{\prime}} \ln \left(\frac{K_{o x}}{K_{s p}^{\prime}}\right)$ 


\subsection{Suggested High-K Gate Dielectric Model and Selected Nano-Materials}

The parasitic capacitances are becoming an important issue for designing logic circuits to reduce MOS transistor dimensions into the deep sub-micrometer regime. Hence, an accurate suggested model in of bottom fringing capacitances is necessary to predict circuit performance before fabrication, thus; the suggested model is an efficient tool in designing and characterization of high-K gate-dielectric SOIMOSFETs including the effects of parasitic internal fringe capacitance [28]. The conventional gate dielectric structure diagram of the TFT devices under study is shown in Figure 2(a), silicon dioxide with dielectric constant $\mathrm{K}_{\mathrm{ox}}=3.9$ has been used as a gate oxide material for decades. The suggested model structure as shown in Figure 2(b) which includes gate dielectric with high-K value for enhancing the performance TFT devices by using novel nanocomposite with high dielectric constant value $\mathrm{K}_{\mathrm{ox}}=25$.

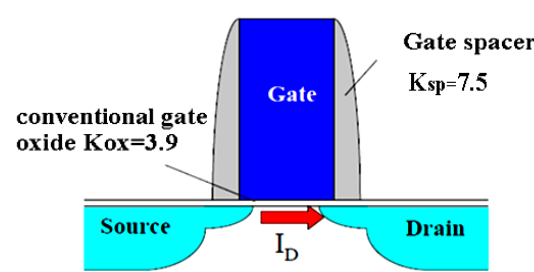

Figure 2(a). Single layer conventional gate dielectric and suggested of gate dielectric TFTs, Conventional gate dielectric oxide

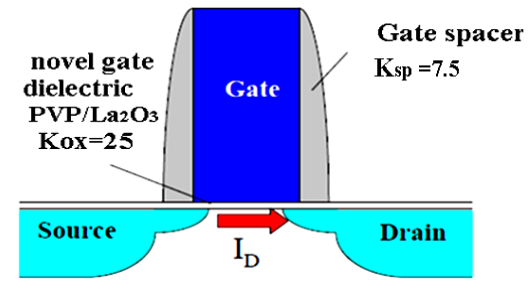

Figure 2(b). Single layer conventional gate dielectric and suggested of gate dielectric TFTs, Novel nanocomposite gate dielectric

The suggested nanocomposite materials have potential candidates for using in a wide range of applications in low cost due to low process temperature of these devices may pave way for achieving high performance for TFT devices. Inserting nanocomposite materials between the gate and S/D contacts are very effective in relaxing the requirements on the virtual source velocity. Lanthanum oxide $\mathrm{La}_{2} \mathrm{O}_{3}$ blended with polyvinyl phenol PVP instead of silicon dioxide. So that, homogeneous organic-inorganic slurry has been obtained, 10wt\% PVP and $\mathrm{La}_{2} \mathrm{O}_{3}$ nano particles, to introduce a gate dielectric nanocomposite gate with high$\mathrm{k}$ dielectric constant and used in the suggested model. According to the mobility degradation is one of the major challenges for MOSFETs with high-K gate dielectrics, this model MOSFETs with PVP/La $\mathrm{O}_{3}$ dielectric film has been solved this problem, as PVP which has been enhanced electron mobility and on-off ratios.

\subsection{Suggested Low-K Gate Sidewall Spacer Model and Selected Nano-Materials}

Estimation of electronic circuit performance is necessary in the design phase to be ensured proper reliability. Thus, fringing capacitance is one of the major contributors to the degradation performance and signal integrity problems in sub-100-nm circuits. Hence, an accurate suggested model of fringing capacitances has been investigated to enhance and predict the circuit performance before fabrication. The conventional gate structure diagram of the TFT devices under study is shown in Figure 3(a) in this structure. Shrinking the conventional MOSFET beyond the $45 \mathrm{~nm}$ technology node requires innovations to suppress parasitic fringing capacitances and short channel effects. Thus, the suggested model structure as shown in Figure 3(b) has been included gate sidewall spacer value enhances the gate control of short-channel effects SCEs.

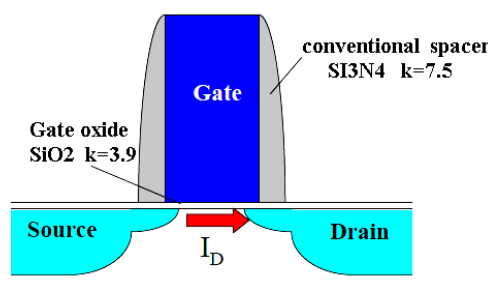

Figure 3(a). A single layer conventional and suggested model of gate spacer TFTs, Conventional gate dielectric spacer

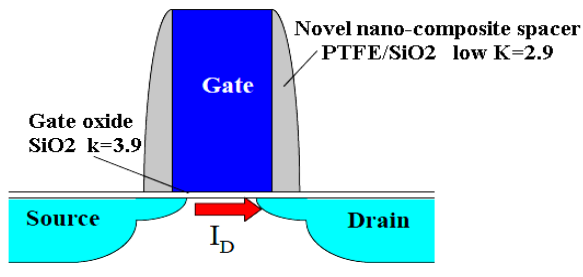

Figure 3(b). A single layer conventional and suggested model of gate spacer TFTs, Novel nanocomposite gate dielectric spacer 
Also, reduces the source-to-drain leakage current and enhances the performance TFTs devices by using spacer with low $\mathrm{K}$ value $\mathrm{K}_{\mathrm{SP}}=2.9$ of Silicon dioxide blended with Poly (tetrafluoroethylene) PTFE instead of silicon nitride in the sidewall spacers. Therefore, inserting nanocomposite materials $\left(20 \mathrm{wt} \% \mathrm{SiO}_{2}\right.$ nano- particles and PTFE) between the gate and S/D contacts is effective in relaxing the requirements on the virtual source velocity and for introducing a gate dielectric spacer with low-K dielectric constant. If silicon dioxide concentration increases up to value of $20 \mathrm{wt} \%$, it may be result in the higher leakage current also structure defects induced by the present of high concentration $\mathrm{SiO}_{2}$.

\subsection{Estimation of Fringing-Capacitance and Surface Potential of Low-K Gate Sidewall Spacer}

From studies cases of MOSFETS [6-16], the fringing capacitances of a low-K gate dielectric are shown in Figure 4, where $\mathrm{C}_{\mathrm{of}}$ is the gate dielectric fringing capacitance, $\mathrm{C}_{\mathrm{gf}}$ is the gate electrode fringing capacitance, $\mathrm{C}_{\mathrm{ox}}$ is the gate dielectric capacitance between the gate and channel, $\mathrm{T}_{\mathrm{ox}}$ and $\mathrm{T}_{\mathrm{g}}$ are the physical thickness of the gate dielectric and gate electrode, respectively, $\mathrm{L}$ and $\mathrm{L}_{\mathrm{a}}$ are channel length and distance between source/drain electrode and gate dielectric respectively, $\mathrm{t}$ is width of overlap region between source/drain and gate electrode, $\mathrm{O}$ is the origin of the $(\mathrm{x}, \mathrm{y})$ coordinate system.

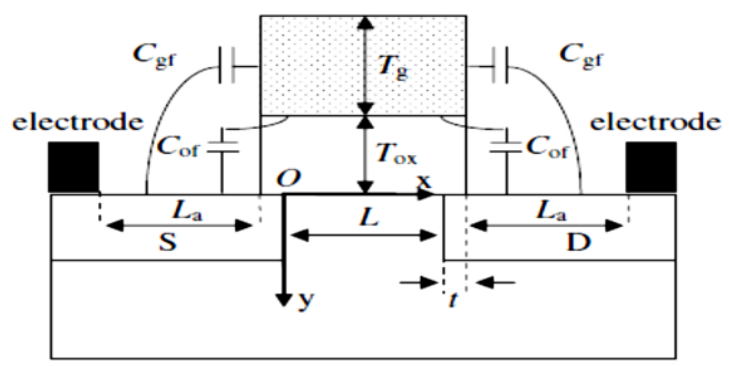

Figure 4. Fringing capacitances for MOSFET

\subsection{Estimation of Surface Potential}

The field lines terminate at the surface of the source/drain regions, leading to an increase of the gate electrode fringing-capacitance. For a given gate dielectric constant, the gate dielectric fringing-capacitance increases with the dielectric constant of the sidewall spacer. It has been that degradation in short-channel performance with high-k dielectrics is caused by the fringing fields from, because the fringing fields can affect the electric potential in the channel, by weakening the gate control [2]. When $\mathrm{V}_{\mathrm{ds}}$ is small, $\mathrm{V}_{\mathrm{d}} \cong \mathrm{V}_{\mathrm{s}}=\mathrm{V}_{\mathrm{bi}}$. Where $V_{s}$ and $V_{d}$ are the source and drain voltages, $V_{b i}$ is built-in potential of the source/substrate and drain/substrate junctions, $\mathrm{V}_{\mathrm{ds}}$ is the potential difference between drain and source. Thus, the induced charge density at the surface of source or drain region by the fringing field lines of $\mathrm{C}_{\text {of }}$ is

$$
\sigma_{\text {of }}=C_{\text {of }} \frac{\left(\mathrm{V}_{\mathrm{G}}-\mathrm{V}_{\mathrm{bi}}\right)}{\mathrm{WL}_{\mathrm{a}}}
$$

Where $V_{G}=V g-V_{f b}$

These charges produce an electric field in the channel, and the relevant surface potential is [7].

$$
\Phi(x, 0)=\frac{\sigma_{\mathrm{of}}}{8 \pi \mathrm{k}_{\mathrm{si}}}\left(\left(\mathrm{x}+\mathrm{L}_{\mathrm{a}}\right) \ln \left(\Phi_{1}\right)-\mathrm{x} \ln \left(\Phi_{1}\right)+\mathrm{W} \ln \left(\Phi_{2}\right)\right)
$$

For the same reason, the surface potential induced by Cgf can be found:

$$
\phi_{\mathrm{gf}}(\mathrm{x}, 0)=\frac{\sigma_{\mathrm{gf}}}{8 \pi \mathrm{k}_{\mathrm{si}}}\left(\left(\mathrm{x}+\mathrm{L}_{\mathrm{a}}\right) \ln \left(\Phi_{1}\right)-\mathrm{x}\left(\Phi_{1}\right)+\mathrm{W} \ln \left(\Phi_{2}\right)\right)
$$


Where:

$$
\begin{aligned}
& \Phi_{1}=\frac{\sqrt{\left(x+L_{a}\right)^{2}+\frac{W^{2}}{4}}+\frac{w}{2}}{\sqrt{\left(x+L_{a}\right)^{2}+\frac{W^{2}}{4}}-\frac{w}{2}} \\
& \Phi_{2}=\frac{\sqrt{x^{2}+\frac{W^{2}}{4}+L_{a}+\sqrt{x^{2}+L_{a}{ }^{2}+2 L_{a} \sqrt{x^{2}+\frac{W^{2}}{4}}}}}{\sqrt{x^{2}+\frac{W^{2}}{4}}+x} \\
& \sigma_{\text {gf }}=\operatorname{Cgf}(V G-V b i) .
\end{aligned}
$$

The electric potential of short channel MOSFET without the influence of the fringing fields was derived to be [11]:

$$
\phi_{\mathrm{s}}(\mathrm{x}, \mathrm{y})=\phi_{0}(\mathrm{y})+\left(\mathrm{V}_{\mathrm{bi}}+\mathrm{V}_{\mathrm{ds}}\right) \frac{\sinh \left(\frac{\mathrm{x}}{\mathrm{I}}\right)}{\sinh \left(\frac{\mathrm{L}}{\mathrm{l}}\right)}+\mathrm{V}_{\mathrm{bi}} \frac{\sinh \left(\frac{\mathrm{L}-\mathrm{x}}{1}\right)}{\sinh \left(\frac{\mathrm{L}}{1}\right)}
$$

Where $\phi_{0}(\mathrm{y})$ is the electric potential of long channel CMOS; $\mathrm{l}=\sqrt{\frac{\mathrm{K}_{\mathrm{si}} \mathrm{y}_{\mathrm{d}}}{\mathrm{C}_{\mathrm{ox}}}}$

CMOS; 1 is a characteristic length describing the short-channel effect; $y_{d}$ is width of the depletion region in the substrate [11]. Considering the influence of the fringing capacitances, the threshold voltage $\mathrm{V}_{\text {th }}$ is defined as the gate voltage when the total surface potential at the location of minimum surface potential $\left(\mathrm{x}_{0}\right)$

$$
\phi_{\mathrm{s}}\left(\mathrm{x}_{0}\right)+\phi_{\mathrm{of}}\left(\mathrm{x}_{0}\right)+\phi_{\mathrm{gf}}\left(\mathrm{x}_{0}\right)=2 \emptyset_{\mathrm{B}}
$$

Where: $\emptyset_{B}=\frac{k_{B} T}{q} \ln \left(\frac{N_{A}}{n_{i}}\right)$ is the Fermi potential of the substrate, when $V_{d s}$ is small, $x_{0} \approx L / 2$. Therefore, the threshold voltage can be found by Eqs. (12) and (14)

$$
V_{t h}=V_{t h 0}-\frac{2\left(V_{b i-} 2 \emptyset_{B}+\phi_{o f}\left(x_{0}\right)+\phi_{g f}\left(x_{0}\right)\right)}{2 \cosh \left(\frac{L}{2 l}\right)-2}
$$

Where, Vtho represents the threshold voltage of long channel MOSFET, and the threshold voltage roll-off increases with $\mathrm{K}_{\mathrm{SP}}$ in the short-channel region.

\section{RESULTS AND DISCUSSION}

This paper has been discussed the following items: enhancing TFTs using high-K gate dielectric nanocomposite material, enhancing TFTs using low-K gate spacer nanocomposite material, and finally the modification of enhancing TFTs using high-K and low-K gate sidewall spacer with nanocomposite material.

\subsection{Enhancing TFTs using High-K Gate Dielectric Nanocomposite Material}

Figure 5 and 6 illustrate the effect of nanocomposite gate dielectric film in internal fringe capacitance $\mathrm{C}_{\text {bottom }}$ by the proposed model. Figure 5 shows the internal fringe capacitance $\mathrm{C}_{\text {bottom }}$ versus the gate spacer dielectric permittivity. It's cleared that fringing capacitance $\mathrm{C}_{\text {bottom }}$ increases with inserting $\mathrm{K}_{\mathrm{ox}}$ by 
a novel nanocomposite material higher than conventional gate dielectric. Figure 6 shows the internal fringing capacitance as a function of normalized gate length for a novel and conventional gate dielectrics. This figure illustrates the fringing capacitance $\mathrm{C}_{\text {bottom }}$ decreases with increasing the gate length from $10 \mathrm{~nm}$ to $80 \mathrm{~nm}$, but $\mathrm{PVP} / \mathrm{La}_{2} \mathrm{O}_{3}$ gives the highest fringing capacitance from conventional gate dielectric with $\mathrm{SiO}_{2}$, at given drain bias $\mathrm{V}_{\mathrm{D}}=0.05 \mathrm{~V}$.

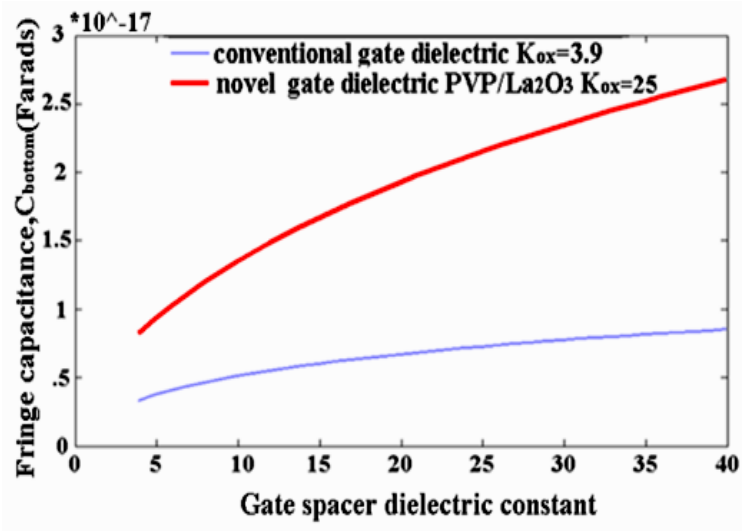

Figure 5. Effect of gate spacer dielectric constant on internal fringing-capacitance $\mathrm{C}_{\text {bottom }}$ using $\mathrm{PVP} / \mathrm{La}_{2} \mathrm{O}_{3}$ and $\mathrm{SiO}_{2}$ gate dielectric $\mathrm{K}_{\mathrm{ox}}$

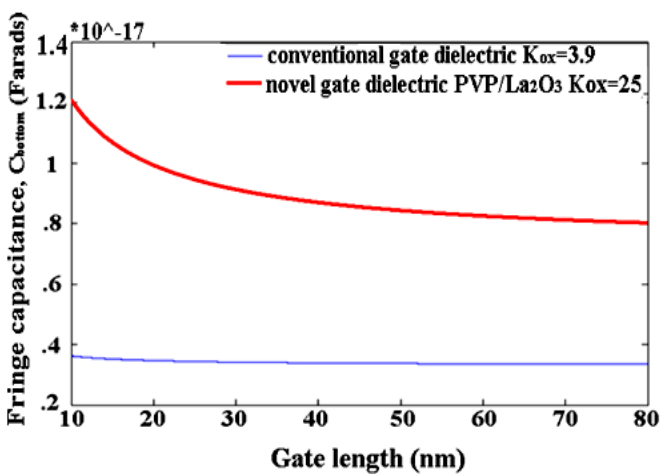

Figure 6 Effect of gate length on internal fringing-capacitance $\mathrm{C}_{\text {bottom }}$ using PVP/La $\mathrm{O}_{3}$ and $\mathrm{SiO}_{2}$ gate dielectric $\mathrm{K}_{\mathrm{ox}}$

Figure 7 and 8 show the effect of nanocomposite high-K gate dielectric film, and parasitic internal fringe capacitance on the surface potential. Figure 7 shows the influence of high-K dielectrics and sidewall spacers on gate surface potential, it's noticed that novel nanocomposite gate dielectric gives higher surface potential than conventional gate dielectric constant with increasing gate spacer dielectric constant. Fig. 8 shows the calculated surface potential variation along the channel for $K_{o x}=25$ with and without the effect of the fringe capacitance. Figure 10 shows the effect of nanocomposite gate dielectric film on the threshold voltage. The novel nanocomposite material of $\mathrm{PVP} / \mathrm{La}_{2} \mathrm{O}_{3}$ has low threshold voltage as compared to those with conventional $\mathrm{SiO}_{2}$ of the same width. Related to high of permittivity of nanocomposite materials, the fringing gate capacitances $\mathrm{C}_{\text {bottom }}$ much high in TFT devices and so, high permittivity of nano composite material increases the surface potential as a result of the induced charges in the drain and source regions due to fringing field lines from the bottom of the gate electrode. Figure 9 explains that the novel nano composite material $\mathrm{PVP} / \mathrm{La}_{2} \mathrm{O}_{3}$ has less threshold voltage as compared with conventional $\mathrm{SiO}_{2}$ of the same width. As a result of high permittivity of nanocomposite materials, the fringing gate capacitances $\mathrm{C}_{\text {bottom }}$ have much high in TFT devices. So, high permittivity of nanocomposite material increases the surface potential of the induced charges in the drain and source regions due to fringing field lines from the bottom of the gate electrode. The drop in the threshold voltage is as high as about $25 \mathrm{mV}$ for $\mathrm{Lg}=60 \mathrm{~nm}$ as cox increases from 3.9 to 25 . All these results are early onset of inversion the channel and hence lower threshold voltage.

Effect of Novel Nanocomposite Materials for Enhancing Performance of Thin Film ... (Youssef Mobarak) 


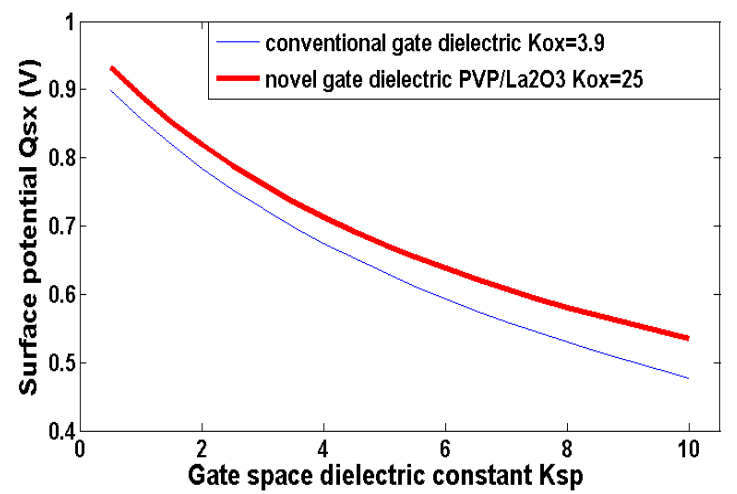

Figure 7. Effect of gate spacer dielectric of nanocomposite high-K gate dielectric film, and parasitic internal fringing capacitance on the surface potential for devices with $\mathrm{SiO}_{2}$ and high $\mathrm{K}_{\mathrm{ox}} \mathrm{PVP} / \mathrm{La}_{2} \mathrm{O}_{3}$ gate dielectric material.

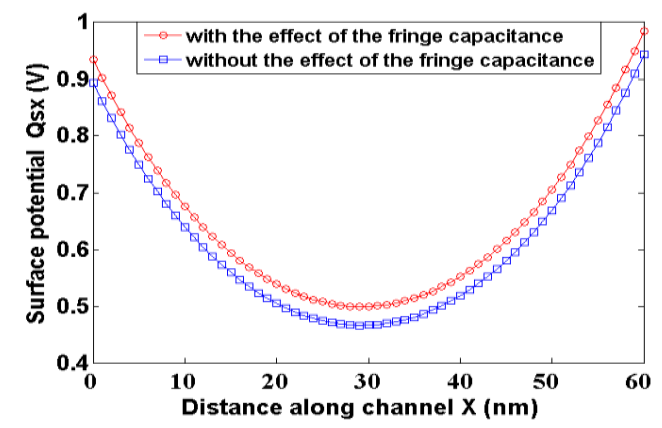

Figure 8. Effect of distance along channel of nanocomposite high-K gate dielectric film, and parasitic internal fringing capacitance on the surface potential for devices with $\mathrm{SiO}_{2}$ and high $\mathrm{K}_{\mathrm{ox}} \mathrm{PVP} / \mathrm{La}_{2} \mathrm{O}_{3}$ gate dielectric material.

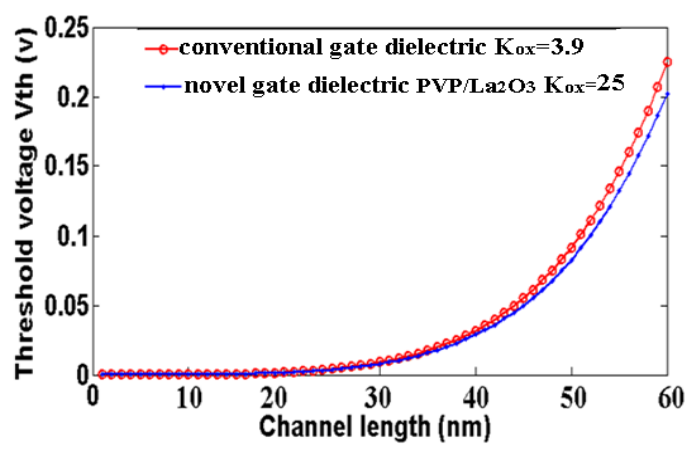

Figure 9. Influence of $\mathrm{K}_{\mathrm{ox}}$ value of gate dielectric film on the threshold voltage of TFT

\subsection{Enhancing TFTs using Low-K Gate Spacer Nanocomposite Material}

Figure 10 and 11 illustrate that the effect of nanocomposite sidewall spacer dielectric film gate in electrode fringing capacitance $\mathrm{C}_{\mathrm{gf}}$. The suggested model indicates that the electrode fringing capacitance $\mathrm{C}_{\mathrm{gf}}$ decreases with increasing $\mathrm{T}_{\mathrm{ox}}$ due to increasing distance between the two plates of $\mathrm{C}_{\mathrm{gf}}$. It is cleared that, the calculated results of the electrode fringing capacitance $\mathrm{C}_{\mathrm{gf}}$ for novel suggested nanocomposite material of side-wall spacer dielectric film gate $\mathrm{PTFE} / \mathrm{SiO}_{2}$ has lower than the conventional side-wall spacer dielectric film gate $\mathrm{Si}_{3} \mathrm{~N}_{4}$ spacer $\mathrm{K}_{\mathrm{sp}}$ as shown in Figure 10. In case of Fig. 11, the variation of the gate electrode fringing- capacitance has been shown with varying gate thickness. For a given EOT and $\mathrm{K}_{\mathrm{ox}}$, the gate electrode fringing-capacitance increases with $\mathrm{T}_{\mathrm{g}}$. Also, it is cleared that, the calculated results of the electrode fringing capacitance $\mathrm{C}_{\mathrm{gf}}$ for novel suggested nanocomposite material of side-wall spacer dielectric film gate 
$\mathrm{PTFE} / \mathrm{SiO}_{2}$ has lower than the conventional side-wall spacer dielectric film gate $\mathrm{Si}_{3} \mathrm{~N}_{4}$ spacer $\mathrm{K}_{\mathrm{sp}}$ jn shown in Figure 11. In case of Figure 12, the effect of nanocomposite sidewall spacer dielectric film has been shown on the gate dielectric fringing-capacitance $\mathrm{C}_{\mathrm{of}}$. It is notices that the gate dielectric fringing-capacitance $\mathrm{C}_{\text {of }}$ by using novel nanocomposite suggested material $\mathrm{PTFE} / \mathrm{SiO}_{2}$ is lower than the gate dielectric fringingcapacitance $\mathrm{C}_{\text {of }}$ with conventional $\mathrm{Si}_{3} \mathrm{~N}_{4}$, this means that the field lines from the bottom of the gate electrode can easily go through the interface between the gate dielectric and sidewall spacer. Figure 13 shows the threshold voltage roll-off $\left(\mathrm{V}_{\text {roll-off }}=\mathrm{V}_{\text {th }}-\mathrm{V}_{\text {tho }}\right)$ for different $\mathrm{K}_{\mathrm{sp}}$ values of sidewall spacer. In case of Fig 13, it has been depicted that the effect of nanocomposite sidewall spacer dielectric film on the threshold voltage of MOSFET degradation in short-channel performance associated with high- $\mathrm{K}_{\mathrm{sp}}$ dielectrics. Thus, it is cleared that replacement of $\mathrm{Si3N} 4$ with $\mathrm{PTFE} / \mathrm{SiO}_{2}$ leads to a significant degradation of the threshold voltage of MOSFET.

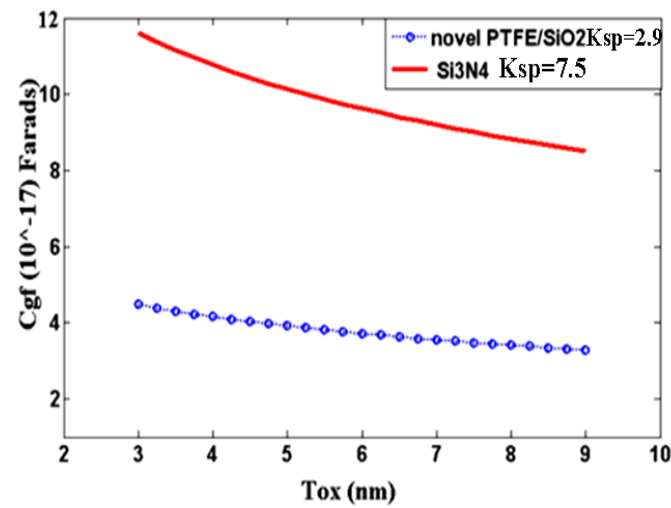

Figure 10. Effect of Gate oxide thickness $\mathrm{T}_{\mathrm{ox}}$ of nanocomposite side-wall spacer dielectric film gate in electrode fringing capacitance $\mathrm{C}_{\mathrm{gf}}$ using $\mathrm{PTFE} / \mathrm{SiO}_{2}$ and $\mathrm{Si}_{3} \mathrm{~N}_{4}$ spacer $\mathrm{K}_{\mathrm{sp}}$

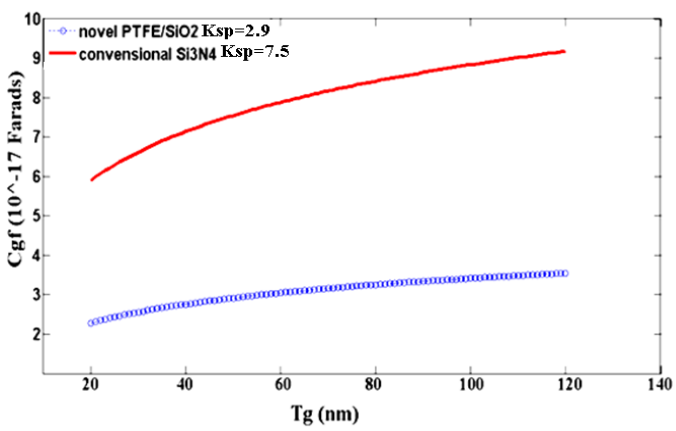

Figure11. Effect of Gate electrode thickness $\mathrm{T}_{\mathrm{g}}$ of nanocomposite side-wall spacer dielectric film gate in electrode fringing capacitance $\mathrm{C}_{\mathrm{gf}}$ using PTFE/SiO 2 and $\mathrm{Si}_{3} \mathrm{~N}_{4}$ spacer $\mathrm{K}_{\mathrm{sp}}$

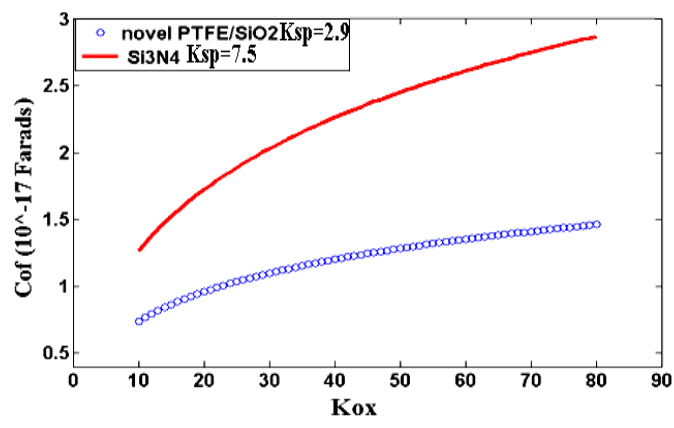

Figure 12. Effect of gate oxide thickness $\mathrm{T}_{\mathrm{ox}}$ in gate dielectric fringing-capacitance $\mathrm{C}_{\text {of }}$ with $\mathrm{PTFE} / \mathrm{SiO}_{2}$ and $\mathrm{Si}_{3} \mathrm{~N}_{4}$ 


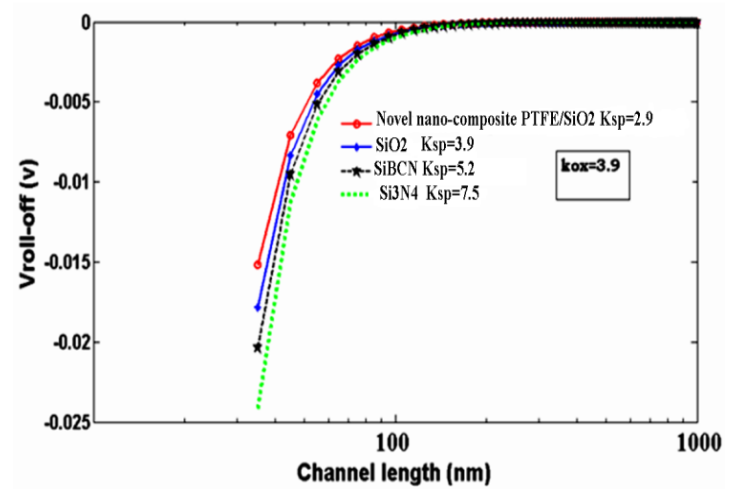

Figure 13. Influence of $\mathrm{K}_{\mathrm{sp}}$ value of sidewall spacer on the threshold voltage of MOSFET

\subsection{Modification of Enhancing TFTs using High-K and Low-K Gate Sidewall Spacer with Nanocomposite Materials}

Figure 14 shows the effect of both novel nanocomposite gate and spacer dielectric film in internal fringe capacitance $\mathrm{C}_{\text {bottom }}$. Figure 14 shows the variation of internal fringing capacitance as a function of normalized gate length for both novel nanocomposite gate dielectric $\mathrm{K}_{\mathrm{ox}} \mathrm{PVP} / \mathrm{La}_{2} \mathrm{O}_{3}$ and gate spacer $\mathrm{K}_{\mathrm{sp}}$ $\mathrm{PTFE} / \mathrm{SiO}_{2}$, with conventional gate dielectrics and gate sidewall spacer dielectric film. This figure shows the fringing capacitance $\mathrm{C}_{\text {bottom }}$ decreases with increasing the gate length but the using of novel nanocomposites give the highest bottom fringing capacitance over conventional materials.

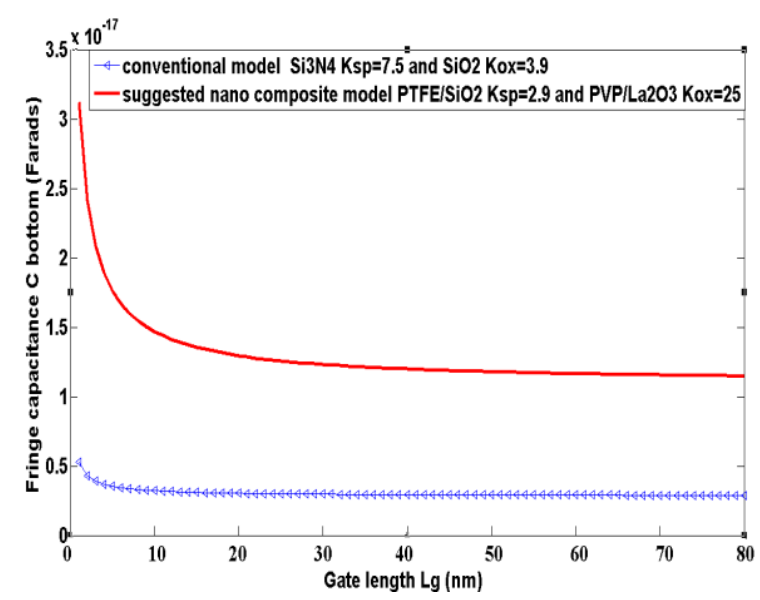

Figure 14. Effect of gate length $\mathrm{L}_{\mathrm{g}}$ on internal fringing-capacitance $\mathrm{C}_{\mathrm{bottom}}$ using novel nanocomposite $\mathrm{PVP} / \mathrm{La}_{2} \mathrm{O}_{3}$ as gate dielectric $\mathrm{K}_{\mathrm{ox}}$ and $\mathrm{PTFE} / \mathrm{SiO}_{2}$ gate sidewall spacer dielectric film $\mathrm{K}_{\mathrm{sp}}$

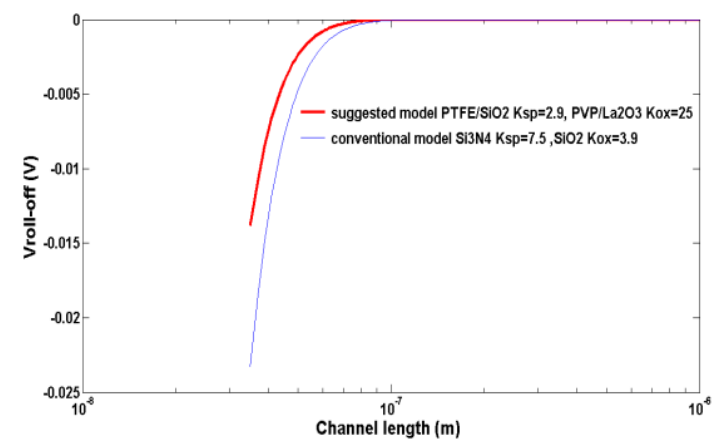

Figure 15. Influence of novel nanocomposite $\mathrm{K}_{\mathrm{ox}}$ and $\mathrm{K}_{\mathrm{sp}}$ on the threshold voltage of MOSFET 
Figure 15 shows the effect of both novel nanocomposite gate and spacer dielectric film in the threshold voltage of MOSFET. It has been cleared that, the threshold voltage roll-off decreases more with using of novel nanocomposite gate dielectric $\mathrm{K}_{\mathrm{ox}} \mathrm{PVP} / \mathrm{La}_{2} \mathrm{O}_{3}$ and gate spacer $\mathrm{K}_{\mathrm{sp}} \mathrm{PTFE} / \mathrm{SiO}_{2}$ than the conventional materiel which used in the short-channel region of $<100 \mathrm{~nm}$ because the FIBL effect is enhanced for large $\mathrm{K}_{\mathrm{sp}}$ values. Therefore, low- $\mathrm{K}_{\mathrm{sp}}$ sidewall spacer can alleviate the FIBL effect, especially for nano MOSFET

\section{CONCLUSIONS}

Highly accurate analytical models have been provided for fringing field effects with the impact of using nanocomposite $\mathrm{PVP} / \mathrm{La}_{2} \mathrm{O}_{3}$ high-K gate dielectrics on device short channel and circuit performance. Novel nanocomposite PTFE/SiO $\mathrm{S}_{2}$ low-K sidewalls spacer has been improved the device short channel performance by reducing the gate fringing field effect. Novel nanocomposite high-K gate dielectric material $\mathrm{PVP} / \mathrm{La}_{2} \mathrm{O}_{3}$ has been improved the performance of TFT devices by increasing the surface potential and decreasing the threshold voltage. High permittivity's $\mathrm{K}_{\mathrm{ox}}$ gate dielectric and greater physical thicknesses, a factor of $\mathrm{K}_{\mathrm{ox}} / \mathrm{KSiO}_{2}$ prevent direct gate tunneling and decreasing gate leakage current.

\section{REFERENCES}

[1] J. U. Duncombe, "Infrared navigation Part I: An assessment of feasibility (Periodical style)," IEEE Trans. Electron Devices, Jan. 1959, vol. ED-11, pp. 3439.

[2] M. Young, TheTechnical Writers Handbook.Mill Valley, CA: University Science, 1989, pp. 1023-1045,

[3] D. Knuth. The Art of Programming. Addison-Wesley, 1973, pp. 82-86.

[4] E. H. Miller, "A note on reflector arrays (Periodical style-Accepted for publication)," IEEE Trans. Antennas Propagat.

[5] S. P. Binguac, "On the compatibility of adaptive controllers (Published Conference Proceedings style)," in Proc. 4th Annu. Allerton Conf. Circuits and Systems Theory, New York, 1994, pp. 8-16.

[6] H.S Momose, M. Ono, and T. Yoshitomi, "1.5 nm direct tunneling gate oxide Si MOSFET's", IEEE electron device letters, 1996, Vol.43, No.8, pp.1233-1242.

[7] M.J. Kumar, S.K. Gupta, and V. Venkataraman, "Compact modeling of the effects of parasitic internal fringe capacitance on the threshold voltage of high-k gate dielectric nano-scale SOI MOSFETs", IEEE TransactionElectron Devices, 2006. Vol.53, No, 4, pp.706-711.

[8] H. Hammer, "Analytical Model for Comb-Capacitance Fringe Fields" journal of micro-electromechanical systems, 2010, Vol.19, No.1, pp.175-182,

[9] M. J. Kumar and A. Chaudhry, "Two-dimensional analytical modeling of fully depleted DMG SOI MOSFET and evidence for diminished SCEs," IEEE Trans. Electron Devices, 2004, Vol. 51, No. 4, pp. 569-574.

[10] C.H. KO, T.M. Kuan, K. Zhang, G. Tsai, S. M. Seutter, C.H. Wu, T.J. Wang, and C.N. Ye, "A Novel CVD-SiBCN Low-K Spacer Technology for High-Speed Applications", Symposium on VLSI Technology Digest of Technical Papers IEEE, 2008, Vol. 978, pp.108-109.

[11] B. Cheng, M. Cao, R. Rao, A. Inani, P.V. Voorde, W.M. Greene, et al., "The impact of high-gate dielectrics and metal gate electrodes on sub-100nm MOSFETs", IEEE electron device letters, 1999, Vol.46, No.7, pp.1537-1544.

[12] R. M.Nihar, P. D.Madhav, G.N. Siva, and V.R. Rao, "Modeling of parasitic capacitances in deep-submicrometer conventional and high-k dielectric MOS transistors", IEEE electron device letters, 2003, Vol.50, No.4, pp.959-966.

[13] K. Suzuki, "Parasitic capacitance of submicrometer MOSFETs," IEEE Trans. Electron Devices, 1999, Vol. 46, No. 9, pp. 1895-1900.

[14] Sh. Oku, T. Nagase, Sh. Nagamatsu, W.Takashima, and K. Kaneto, "Comparative Study on Gate Insulators of Polymers and $\mathrm{SiO} 2$ in Transport Properties of p- and n-Type Organic Field-Effect Transistors", Japanese journal of applied physics, 2010, Vol. 49.

[15] D. Rébiscoul, S. Favier, J-P. Barnes, J.W. Maes, F. Martin, "Characterization of the annealing impact on La2O3/HfO2 and HfO2/La2O3 stacks for MOS applications", Elsevier journal Microelectronic Engineering, 2010, Vol. 87, pp. 278-281.

[16] B. Aditya, C. P. Bipul, R. Kaushik. "Modeling and optimization of fringing-capacitance of nanoscale DGMOS devices", IEEE electron device letters, 2005, Vol.52, pp.256-262.

[17] X. Li, R.J.W. Hill, H. Zhou, C.D.W. Wilkinson, I.G. Thayne, "A low damage Si3N4 sidewall spacer process for self-aligned sub-100nm III-V MOSFETs" Elsevier Microelectronic Engineering, 2008, Vol .85, pp.996-999.

[18] M. J. Deen, M. H. Kazemeini, and S. Holdcroft, "Contact effects and extraction of intrinsic parameters in poly(3alkylthiophene) thin-film field-effect transistors," J. Appl. Phys., , Jun. 2008, vol. 103, no. 12, p. 124509.

[19] A. Cerdeira, M. Estrada, R. Garcia, A. Ortiz-Conde, and F. Sanchez, "New procedure for the extraction of basic aSi:H TFT model parametersin the linear and saturation regions," Solid State Electron., Jul. 2001, vol. 45, no. 7, pp. $1077-1080$.

[20] M. Hamilton, S. Martin, and J. Kanicki, "Field-effect mobility of organic polymer thin-film transistors," Chem. Mater., 2004, vol. 16, no. 23, pp. 4699-4704.

[21] B. Hamadani and D. Natelson, "Nonlinear charge injection in organic field-effect transistors," J. Appl. Phys., Mar. 2005, vol. 97, no. 6, pp. 064508-1-064508-7. 
[22] H. Klauk, U. Zschieschang, andM. Halik, "Low-voltage organic thin-filmtransistors with large transconductance," J. Appl. Phys., Oct. 2007vol. 102, no. 7,pp. 074514-1-074514-7.

[23] T. Yamamoto and K. Takimiya, "Facile synthesis of highly $\pi$-extended heteroarenes, dinaphtho[2,3-b:2_,3_f]chalcogenopheno[3,2-b]chalcogenophenes, and their application to field-effect transistors,"J. Amer. Chem. Soc., Feb. 2007, vol. 129, no. 8, pp. 2224-2225.

[24] Y. Tsividis, Operation and Modeling of the MOS Transistor, 2nd ed. New York: McGraw-Hill, 1999.

[25] C. Enz and E. Vittoz, "MOS transistor modeling for low-voltageand low-power analog IC design," Microelectron. Eng., Dec. 1997 vol. 39, no. 1-4,pp. 59-76.

[26] M. Shur and M. Hack, "Physics of amorphous silicon based alloyfield-effect transistors," J. Appl. Phys., May 1984, vol. 55 , no. 10 , pp. 3831-3842.

[27] M. J. Deen and M. Kazemeini, "Photosensitive polymer thin-filmFETs based on poly(3-octylthiophene),” Proc. IEEE, , Jul. 2005, vol. 93, no. 7,pp. 1312-1320.

[28] A. Thabet, M. Atef, and Y. A. Mobarak, "Enhanced TFTs Model Using Novel Nano-Composite High-K Gate Dielectric" " Published in Power Electronics and Applications (EPE 2011), Proceedings of the 2011-14th European Conference on 2011, pp. $1-3$.

\section{BIOGRAPHIES OF AUTHOR}

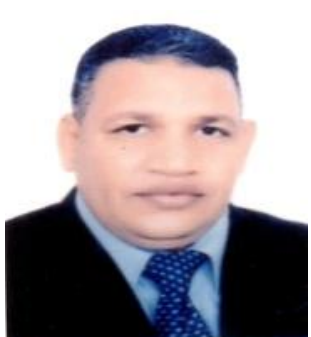

Youssef A. Mobarak was born in Luxor, Egypt in 1971. He received his B.Sc. and M.Sc. degrees in Electrical Engineering from Faculty of Energy Engineering, Aswan University, Egypt, in 1997 and 2001 respectively and Ph.D. from Faculty of Engineering, Cairo University, Egypt, in 2005. He joined Electrical Engineering Department, Faculty of Energy Engineering, Aswan University as a Demonstrator, as an Assistant Lecturer, and as an Assistant Professor during the periods of 1998-2001, 2001-2005, and 2005-2009 respectively. He joined Artificial Complex Systems, Hiroshima University, Japan as a Researcher 2007-2008. Also, he joined Faculty of Engineering, King Abdulaziz University, Rabigh, Saudi Arabia as Associate Professor Position at April 2014 up to date. His research interests are power system planning, operation, optimization, and techniques applied to power systems. Also, his research interests are wind energy, and nanotechnology materials via addition nano-scale particles and additives for usage in industrial field.

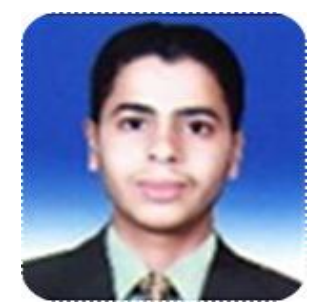

Moamen Atef, He received his B.Sc. degrees in Electrical Engineering from High Institute of Energy, South Valley University, Egypt, in 2009. His research interests lie in the areas of analysis and developing electrical engineering models and applications, investigating novel nano-technology materials via addition nano-scale particles and additives for usage in industrial branch, electromagnetic materials, electroluminescence and the relationship with electrical and thermal ageing of industrial polymers. E-mail : eng.moamenpower@yahoo.com 